\title{
Drought Stress Exacerbates Fungal Colonization and Endodermal Invasion and Dampens Defense Responses to Increase Dry Root Rot in Chickpea
}

\author{
Vadivelmurugan Irulappan, Manu Kandpal, Kumud Saini, Avanish Rai, Aashish Ranjan, Senjuti Sinharoy, \\ and Muthappa Senthil-Kumar ${ }^{\dagger}$
}

National Institute of Plant Genome Research, Aruna Asaf Ali Marg, PO Box No. 10531, New Delhi, India

Accepted 28 February 2022.

\begin{abstract}
Drought plays a central role in increasing the incidence and severity of dry root rot (DRR) disease in chickpea. This is an economically devastating disease, compromising chickpea yields particularly severely in recent years due to erratic rainfall patterns. Macrophomina phaseolina (formerly Rhizoctonia bataticola) is the causal agent of DRR disease in the chickpea plant. The infection pattern in chickpea roots under wellwatered conditions and drought stress are poorly understood at present. This study provides detailed disease symptomatology and the characteristics of DRR fungus at morphological and molecular levels. Using microscopy techniques, the infection pattern of DRR fungus in susceptible chickpea roots was investigated under well-watered and drought-stress conditions. Our observations suggested that drought stress intensifies the progression of already ongoing infection by weakening the endodermal barrier and overall defense. Transcriptomic analysis suggested that the plant's innate immune defense program is downregulated in infected roots when subjected to drought stress. Furthermore, genes involved in hormonal regulation are differentially expressed under drought stress. These findings provide hints in terms of potential chickpea genes to target in crop improvement programs to develop climate-change-resilient cultivars.
\end{abstract}

Keywords: abiotic stress, chickpea, combined stress, drought, dry root rot, fungus-plant interactions, Macrophomina phaseolina, mechanisms of pathogenicity, microscopy and imaging, plant responses to pathogens

Drought stress aggravates dry root rot (DRR) disease in chickpea (Sharma and Pande 2013; Sinha et al. 2019, 2021). It increases the disease incidence and severity in plants experiencing

${ }^{\dagger}$ Corresponding author: M. Senthil-Kumar; skmuthappa@nipgr.ac.in

Funding: This project of the M. Senthil-Kumar lab was supported by core funding from the National Institute of Plant Genome Research and V. Irulappan was supported by research fellowship DBT-JRF/DBT/2015/ NIPGR/430 from the Department of Biotechnology (DBT), India.

*The $\boldsymbol{e}$-Xtra logo stands for "electronic extra" and indicates that a supplementary video, supplementary figures, and supplementary files are published online.

The author(s) declare no conflict of interest.

(c) (1) () $\odot$ Copyright () 2022 The Author(s). This is an open access article distributed under the CC BY-NC-ND 4.0 International license. drought stress compared with well-watered plants (Chandran et al. 2021; Sharma and Pande 2013; Sinha et al. 2019). Drought increases DRR severity regardless of soil type, geography, and genotype (Sinha et al. 2019; 2021). Drought alone causes 40 to $50 \%$ yield loss in chickpea (Maqbool et al. 2017) and, together with DRR disease, even up to $100 \%$ yield loss can occur (Ghosh et al. 2013; Nene et al. 1981; Sinha et al. 2019). Macrophomina phaseolina (Tassi) Goid. (asexual stage, formerly referred to as Rhizoctonia bataticola (Taubenh.) E. J. Butler) is the causal agent of DRR disease. M. phaseolina, a soilborne fungus, is a necrotrophic pathogen. Microsclerotia and mycelia act as the primary inoculum. DRR symptoms usually appear at the reproductive stage under field conditions (Sharma and Pande 2013; Sinha et al. 2019). Symptoms include prematurely dried plants with strawcolored foliage; dry, rotten tap roots devoid of lateral roots; and minute microsclerotia in the epidermis, cortex, and pith regions. In the literature, sick plot, sick pot, and blotting paper techniques have been used to assess the response of chickpea plants to DRR (Irulappan and Senthil-Kumar 2021; Irulappan et al. 2021). Despite the proven inheritance of DRR resistance (Rao and Haware 1987; Talekar et al. 2017), identification of quantitative trait loci associated with resistance genes, and genotype screening of genetically diverse germplasm accessions (Infantino et al. 2006; Karadi et al. 2021; Talekar et al. 2017), no headway in DRR resistance breeding has been made. This gap can be attributed to the lack of understanding of the fungal morphology, taxonomy, and disease cycle of the causal fungal pathogen and plant responses to the fungus at pathomorphological and molecular levels under well-watered and drought-stress conditions. Specifically, systematic studies on fungal infection stages during interaction with the chickpea root are warranted. The present study aimed to elucidate the mechanistic details of drought-induced plant susceptibility to DRR and underlying differential gene expression associated with susceptibility.

\section{RESULTS AND DISCUSSION}

A highly virulent DRR fungal isolate was isolated from fieldgrown chickpea plants showing typical DRR symptoms such as easily uprooted plants leaving most of the rotten primary root containing microsclerotia in the soil (Supplementary Figs. S1 and S2). Internal transcribed spacer segment sequencing and dendrogram construction confirmed the fungal identity (Supplementary Fig. S2). On potato dextrose agar medium, we observed septate, multinucleate mycelia with right-angled branching. The septate mycelia and microsclerotia served as the primary inoculum for the disease cycle (Supplementary Figs. S3 to S6). Hyphal fusion was observed in the fungus interacting with roots (Supplementary Fig. S5F). Systematic confocal microscopy investigation on initial 
infection and colonization in the cortex, endodermis, and pith regions of the pathogen-inoculated root was performed on fungus labeled with chitin-specific fluorescein isothiocyanate-conjugated wheat germ agglutinin (WGA-FITC) using a blotting paper technique, which facilitates quick infection progression (Ayliffe et al. 2013; Irulappan and Senthil-Kumar 2021) (Supplementary Figs. S7 to S10). The blotting paper technique entails inoculating chickpea seedlings with the fungal inoculum and monitoring infection progression on blotting paper (Irulappan and Senthil-Kumar 2021). Microsclerotial attachment, mycelial growth, colonization at the cortex, hyphal branching, and formation of daughter microsclerotia were observed during the root infection (Supplementary Fig. S11). The attached microsclerotia (Fig. 1A) gave rise to a germ tube-like structure (Fig. 1B), and mild local necrosis of the epidermal cells was sporadically observed under well-watered conditions. During the initial infection (1 to 4 days after infection [DAI]), the fungus colonized the first two layers of cortical cells (Fig. 1C). At 4 to $5 \mathrm{DAI}$, fungal colonization extended to a large number of cortical cells, with hyphal branching (Fig. 1B to D). Between 6 and 10 DAI, all of the cortical cell layers were colonized by fungal mycelia (Fig. 1E). At the advanced stage of infection, daughter microsclerotia were formed at 12 to $13 \mathrm{DAI}$ (Fig. 1F; Supplementary Fig. S10A to H). Furthermore, upon infection of chickpea roots transformed with an apoplastic localization marker construct, developed using the hairy root transformation technique (Mandal and Sinharoy 2019), the infection progressed intracellularly at early stages of infection (Fig. 1G to I; Supplementary Fig. S12A to D; Supplementary Video S1) and both intra- and intercellularly at later stages of infection (Supplementary Fig. S12E; Supplementary Video S1). Furthermore, confirming the above confocal microscopy observations, scanning electron micrographs also revealed microsclerotial attachment on the root epidermis, followed by the emergence of a germ-tube-like structure, mycelial growth, formation of appressorium-like structures, and penetration into the root epidermis (Fig. 1J to O). Although the fungus is reported to be necrotrophic, barring a few sporadic necrosislike spots on the root epidermis, the observations showed a long biotrophic phase during the initial infection period (Supplementary Fig. S11E to $\mathrm{H}$ ), and such changes are consistent with an earlier report on another similar fungus (Chowdhury et al. 2017). However, at the final stages of infection, typical necrosis was observed, culminating in complete root damage due to the death of diseased cells. Notably, hyphal branching was noticed before the formation of daughter microsclerotia during the initial infection process (Fig. 1P). Estimation of fungal biomass by DNA quantification also confirmed the extent of fungal colonization (Fig. 1Q). These morphopathological observations suggest that infection progression is spatiotemporal in susceptible chickpea roots. In addition, under well-watered conditions, infection progression was arrested at the endodermal layer and did not proceed beyond this layer. The complex nature of the endodermis (namely, the presence of Casparian strips and suberin deposition) might underlie the infection arrest (Geldner 2013). A modified blotting paper technique was used to investigate how drought stress aggravated DRR fungal infection progression (Irulappan and Senthil-Kumar 2021). The experiment was carried out with a control (no treatment) and drought, pathogen, and drought-plus-pathogen (DP) treatments (Supplementary Fig. S13A). Roots from the control and drought treatments did not have any fungal infection. At $9 \mathrm{DAI}$, the pathogen-infected root had less fungal proliferation, and half of the cortex layers were colonized with a smaller number of mycelia. However, pathogeninfected roots subjected to drought stress had high fungal proliferation, because the entire cortex was colonized with a large number of branched hyphae. Correspondingly, at $13 \mathrm{DAI}$, the pathogen under well-watered condition demonstrated fungal colonization up to the cortex cell layer, with a smaller number of hyphae compared with infected roots under drought stress (Fig. 2A to I).
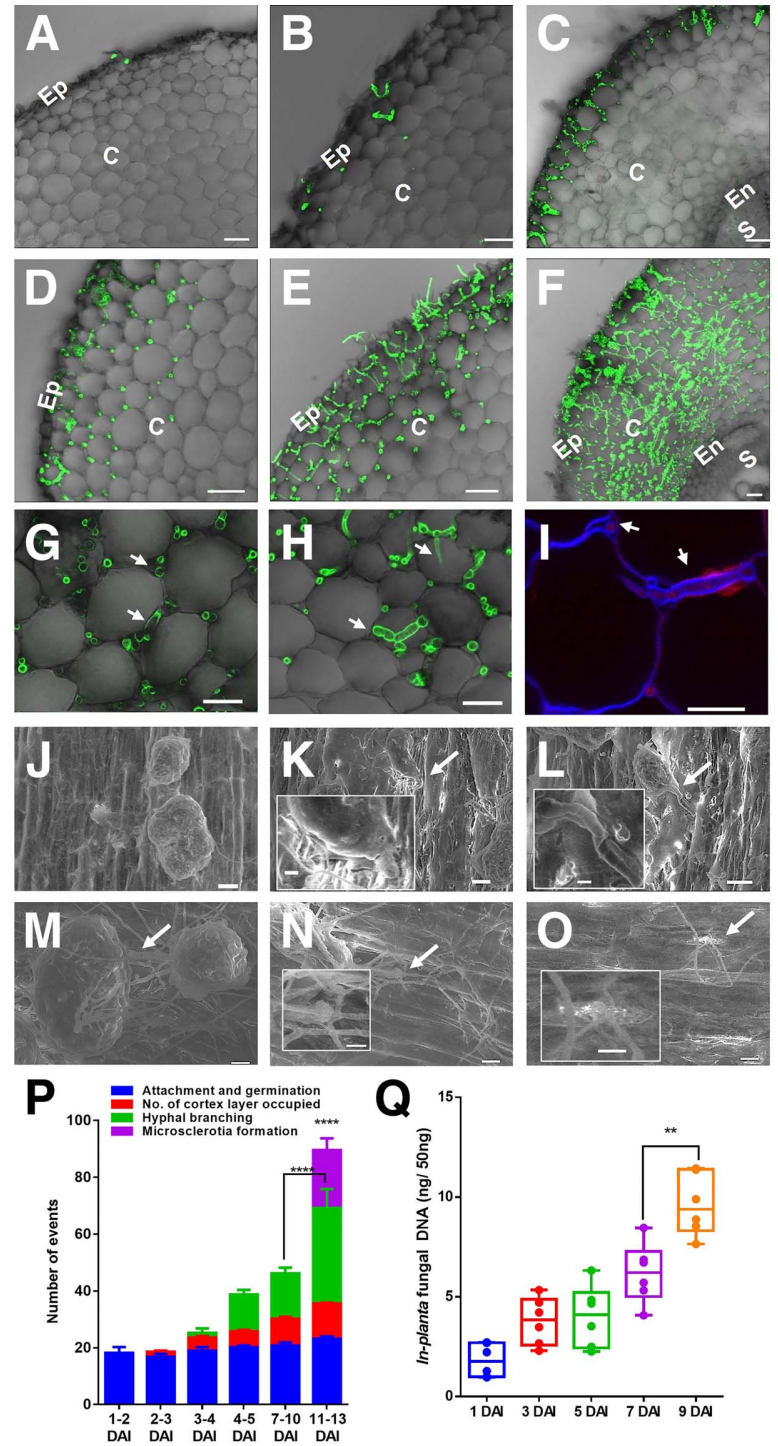

Fig. 1. Dry root rot fungus infection pattern in chickpea root. Transverse sections of fungal-infected chickpea root samples (genotype JG 62) using a modified blotting paper technique were observed for fungal cellular movement under well-watered conditions. A, Attachment of microsclerotia on the root epidermis (1 to 2 days after infection [DAI]). B, Emergence of the germ tube (2 to 3 DAI). C, Mycelial growth and initial colonization of cortex cells (3 to $5 \mathrm{DAI})$. D, Intercellular movement of mycelia via the apoplast (5 to 6 DAI). E, Hyphal branching after colonization of the cortex (7 to 9 DAI). F, Formation of microsclerotia in the cortex (12 to $13 \mathrm{DAI})$. G, Intercellular movement of fungal mycelia. H, Intracellular movement of fungal mycelia. I, Mycelial movement via the apoplastic space labeled with BCPspmCherry (Ivanov and Harrison 2014). Fungal mycelia and microsclerotia are stained green. $\mathbf{J}$ to $\mathbf{O}$, Scanning electron microscopy images showing microsclerotial attachment (1 DAI), germ tube emergence (3 DAI), mycelial emergence (3 DAI), mycelial growth (5 DAI), mycelial extension, appressorium formation (5 DAI), and mycelial penetration into plant epidermis cells (5 DAI). P, Graph showing the number of critical events that occurred during the infection at branching zone 1 at different time points. Q, Graph showing the amount of in planta fungal DNA in the infected roots at different time points. Chickpea root sections were stained with fluorescein isothiocyanateconjugated wheat germ agglutinin (A to $\mathrm{H}$ ) and hairy roots expressing BCPspmCherry (red) were stained with cell-wall-specific calcofluor white (blue) (I). Scale bars: $50 \mu \mathrm{m}$ (A to G), $20 \mu \mathrm{m}$ (H to J and L to P), and $50 \mu \mathrm{m}$ (K). Similar observations were found in at least two independent experiments. $\mathrm{Ep}=$ epidermis, $\mathrm{C}=$ cortex, $\mathrm{En}=$ endodermis, and $\mathrm{S}=$ stele. Experiments $(\mathrm{JG}$ 62 genotype) were repeated at least twice, with five biological replicates, and analyzed by one- and two-way analysis of variance (ANOVA); $n=5$, error bar in the graphs $=$ standard error of the mean. Asterisks show significance: $* * * *$ and ${ }^{* *}$ indicate $P \leq 0.0001$ and 0.01 , respectively, according to Tukey's honestly significant difference and Holm-Sidak's multiple comparisons test after one- and two-way ANOVA. 
As a root pathogen, DRR fungus is expected to colonize the stele region to access nutrients and extend hyphal growth. To further access water and ions, the barrier formed by the endodermal layer must be crossed by extending hyphal colonization (Barberon 2017). However, the Casparian strip and suberin are involved as a barrier against the invasion of root pathogens. The Casparian strip is majorly involved in the regulation of water and ion movement into the stele. Plant roots without the Casparian strip are reported to have high fungal colonization in the vascular region (Fröschel et al. 2021). Similarly, a reduction in suberin, a bidirectional transcellular barrier, in the secondary cell wall has been reported in response to pathogen infection, contributing to plant susceptibility (Barberon 2017; Fröschel et al. 2021). Under well-watered conditions, fungal infection progression was restricted at the endodermal cell layer (Fig. 2G; Supplementary Fig. S13H; Supplementary Video S1), with pathogen-induced hyperplasia at the endodermal or pericycle region. Hyperplasia further tightens the apoplastic intercellular junction in several cell layers around the site of the endodermal barrier, thereby preventing fungal colonization (Supplementary Fig. S14C). To know the pathogen-infection induced cell layer multiplication of pericycle at the site of infection, the number of cell layers was measured at 9 and 13 DAI (Fig. 2J). Although the number of cell layers increased under pathogen-only treatment compared with control at 13 DAI, such an increase was not detected at 9 DAI. However, under DP treatment at $13 \mathrm{DAI}$, the cell layers could not be counted due to disease cell death (Fig. 2J). It is possible that, under well-watered conditions, as a part of plant defense against root pathogens, the Casparian strip, suberin lamellae, and hyperplasia in the endodermis are strengthened in response to infection, which impedes the extension of fungal colonization. However, detailed microscopy across various time points after infection is necessary to state the involvement of hyperplasia conclusively.

When pathogen-infected roots were subjected to drought stress, roots showed fungal colonization beyond the endodermis; namely, in the xylem, phloem, and pith regions (Fig. 2H; Supplementary Fig. S13I). Weakening of the endodermal layer was observed in infected roots under drought stress (Supplementary Fig. S13J and K). Drought stress altered the intercellular apoplastic space in the infected root and, hence, could have facilitated fungal penetration into the endodermis layer (Supplementary Video S1). Hypertrophy in the endodermis cell layer was observed in the infected roots subjected to drought stress, and roots with hypertrophy had extensive colonization in the stele region (Supplementary Fig. S13K). Along with cell expansion, a necrotized endodermis layer was also seen; this necrosis might be due to increased secretion of cell-wall-degrading enzymes by the pathogen (Supplementary Fig. S13K; Supplementary Video S1). We speculate that DRR fungus undermines the plant's innate defense and alters suberin and lignin deposition in the Casparian strip in the endodermis in drought-stressed roots. Taken together, the above observations indicate that systemic fungal spread across the endodermal layer of roots, promoted by drought stress, results in extensive root damage and contributes to aggravated cell death and DRR disease.

Next, gene expression levels were quantified by RNAsequencing (RNA-seq) analysis of chickpea roots subjected to drought, pathogen, and DP stress at two time points (3 and 6 days after sowing [DAS]). A precise sick pot technique, which mimics the natural infection, was used to impose infection and drought stress to study the infection and drought influence at early time points at root branching zone 1 (Supplementary Figs. S15 to S18). The sick pot technique entails growing the chickpea plants in soil or potting medium admixed with the fungus. To study the regulation of different gene families, we expanded our analysis to all transcripts that are upregulated and downregulated by simultaneous pathogen and drought stress. At 6 DAS, 7,510 genes were differentially expressed (2,634 upregulated and 4,876 downregulated) in drought, pathogen, and DP stress conditions at a $1 \%$ false discovery rate (Fig. 3A). The number of differentially expressed genes (DEGs) was higher at 6 than at 3 DAS (Fig. 3A; Supplementary Figs. S19 and S20). Such a shift in the number of DEGs from 3 to 6 DAS might be due to the DRR fungus switching from the early biotrophic phase to the necrotrophic phase in chickpea roots. At 6 DAS, Kyoto Encyclopedia of Genes and Genomes (KEGG) enrichment analysis revealed that upregulated and downregulated features were associated with 9 and 21 biological processes, respectively (Fig. 3B). Downregulated features belonged to processes involved in specialized metabolism (flavonoids, phenylpropanoids, and terpenoids), plant defense, cell wall, signal transduction, hormone signaling pathways (jasmonic acid [JA] and ethylene [ET]), and photosynthesis. Upregulated features were associated with galactose metabolism, cell death, glutathione metabolism, and plant hormone signal transduction. The pathogen negatively modulates photosynthesis and carbohydrate metabolism, which imposes stress on plants and induces switching to defense metabolism. Moreover, the downregulated feature for amino acid and nucleotide sugar metabolism (cell wall biosynthesis) and phenylpropanoid biosynthesis (coumarins, lignin, and suberin) under combined DP stress at 6 DAS indicates the importance of these metabolic pathways in making chickpea roots more susceptible to DRR fungus under drought stress. When comparing drought, pathogen, and DP stress samples from both 3 and 6 DAS, the variation depicted in principal component analysis (PCA) showed the clear separation of samples at two different time points (Supplementary Fig. S20D). However, a marked separation of DP samples at 6 DAS was observed compared with other samples at 6 and 3 DAS (Supplementary Fig. S20C). Furthermore, weighted gene coexpression network analysis (WGCNA) was executed on the well-enriched chickpea expression datasets of drought, pathogen, and DP stress samples at 3 and 6 DAS. WGCNA generated a network comprising 23 different clusters of coexpressed transcripts (Supplemental Figs. S21 to S23). Three different clusters containing differentially regulated and functionally coexpressed transcripts in pathogen and DP stress conditions were selected for further analysis (Fig. 3B). The clusters were identified by coupling DEG testing with KEGG enrichment analyses of gene ontology (GO) terms such as plant hormone signal transduction, plantpathogen interaction, specialized metabolism, defense pathways, and transcriptional regulators. When comparing different selected clusters, the importance of KEGG functional enrichments was validated. The three clusters of genes differed across drought, pathogen, and DP stress at 6 DAS. Cluster 1 comprised mostly downregulated genes in the DP condition at 6 DAS, whereas cluster 3 contained a set of genes that were mostly upregulated in DP at 6 DAS. However, in cluster 2, most of the genes were upregulated in the pathogen-only condition and downregulated under DP stress at 6 DAS compared with the other treatments (Fig. 3C; Supplementary File S1). Several genes associated with plant immunity, terpenoids, and phenylpropanoids fell into clusters 1 and 2. In contrast, the majority of genes underlying growth, cell division, cell damage, oxidative stress, and signal transduction were enriched in cluster 3. In cluster 1, genes involved in JA and ET signaling pathways were found to be downregulated at all stress conditions at 6 DAS but significantly downregulated under DP stress at 6 DAS. Jasmonate ZIM domain (JAZ) proteins belonging to the TIFY family clustered together with the APETALA2/ETHYLENE RESPONSIVE FACTOR (AP2/ERF) family of transcription factors (Fig. 4A; Supplementary File S1). At 3 DAS, the transcript levels of TIFY10A (JAZ10), TIFY5A (JAZ5), TIFY10A (JAZ9), TIFY6B (JAZ6), ETS2 repressor factor 113 (ERF113), ERF110, and ERF028 were significantly upregulated under pathogen stress and downregulated under DP stress. The reduced transcript levels of defense genes under DP stress indicate 

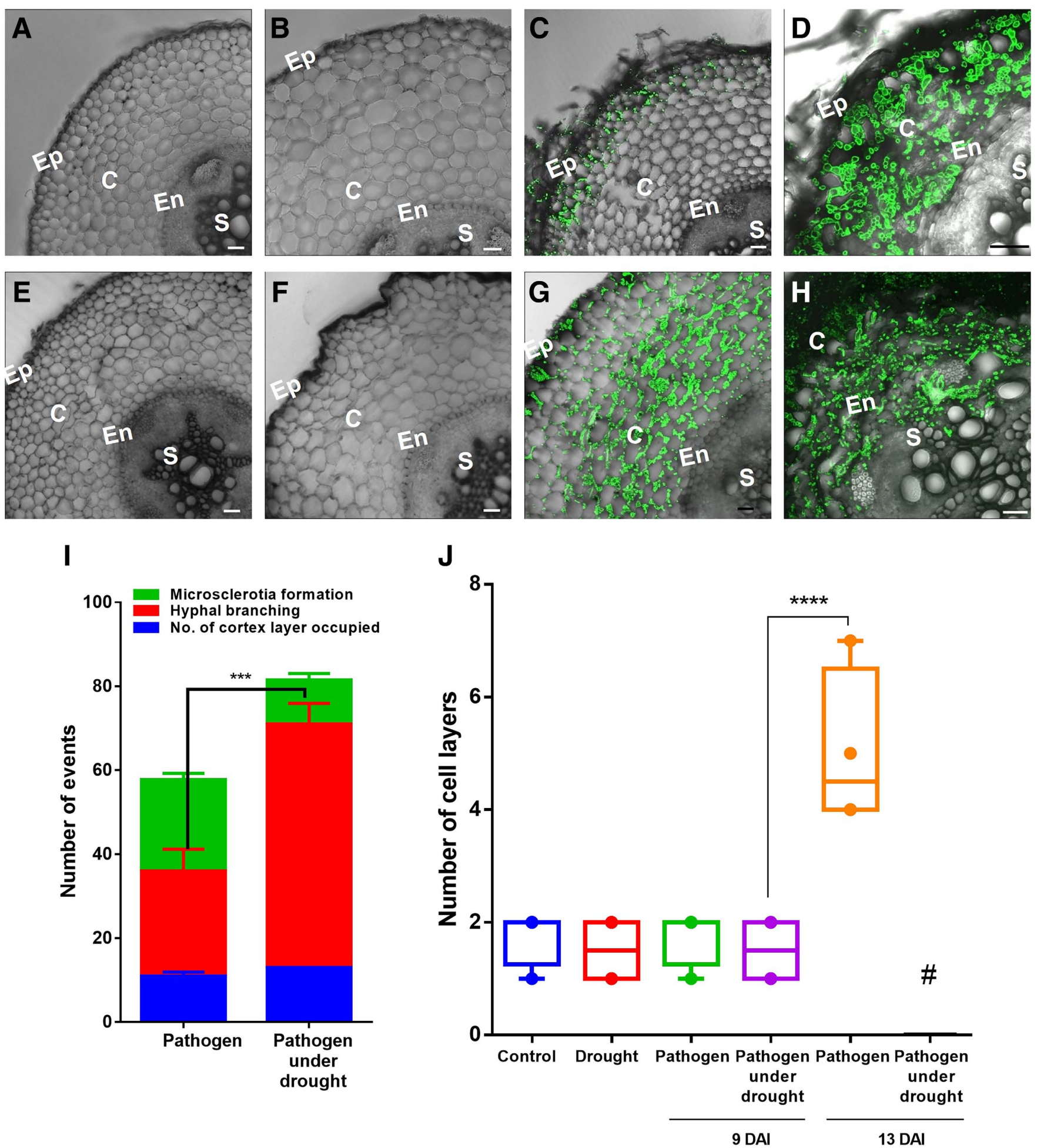

Fig. 2. Drought aggravates dry root rot infection progression. Root samples were collected from control, drought, pathogen, and pathogen-plus-drought treatments from a modified blotting paper technique. Transverse sections of fungal infected roots: A, control (9 days after transfer to the blot); B, drought ( 9 days after transfer to the blot); C, pathogen ( 9 days after infection [DAI]); D, pathogen plus drought (9 DAI); E, control (13 days after transfer to the blot); F, drought (13 days after transfer to the blot); G, pathogen (13 DAI); and $\mathbf{H}$, pathogen plus drought (13 DAI). Fungal mycelia and microsclerotia are stained green. I, Graph showing the number of crucial events that occurred under two different treatments at 13 DAI. J, Graph showing the pathogen-infection-induced cell layer multiplication of endodermis or pericycle at the site of infection (control and drought, 13 days after transfer to the blot). Symbol \# indicates that, due to extensive infection under drought stress conditions, tissue was severely damaged. Hence, the multiplication of cell layers could not be measured. Scale bar $=50 \mu \mathrm{m}$. Similar observations were seen in at least two independent experiments. Ep $=$ epidermis, $\mathrm{C}=$ cortex, $\mathrm{En}=$ endodermis, and $\mathrm{S}=$ stele. Experiments $(\mathrm{JG} 62$ genotype) were repeated at least twice, with five biological replicates, and analyzed by two-way analysis of variance (ANOVA); $n=4$, error bar in the graphs $=$ standard error of the mean. Asterisks show the significance: $* * *$ and $* * * *$ indicate $P \leq 0.0004$ and 0.001 , respectively, according to Tukey's honestly significant difference and Holm-Sidak's multiple comparisons test after one- and two-way ANOVA. 
the suppression of immunity. Moreover, a substantial reduction in the transcript level of genes related to signal transduction (e.g., ETR1/EIN1, WRKY29, and CNGC2) was noticed in infected roots under drought stress (Fig. 4B). ETRI and EIN1 genes are involved in ethylene signaling, which acts against necrotrophic pathogens (Kieber 1997). The subtractive and combinatorial expression of JAZ and ERF proteins determines disease progression under drought stress. In contrast, most of the genes belonging to the auxin and abscisic acid pathway, coexpressed together in cluster 3, were significantly upregulated under DP stress at 6 DAS. Most of the genes belonging to this category have been identified to be involved in growth and development. This implies their principal role in the growth-defense trade-off (Fig. 4A). Metabolic pathways associated with the production of defensive metabolites (mono-, di-, tri, and sesquiterpenes) are clearly coexpressed in different stress conditions (Supplementary Fig. S24). For instance, at 3 and 6 DAS, most of the genes belonging to this category showed reduced transcript levels

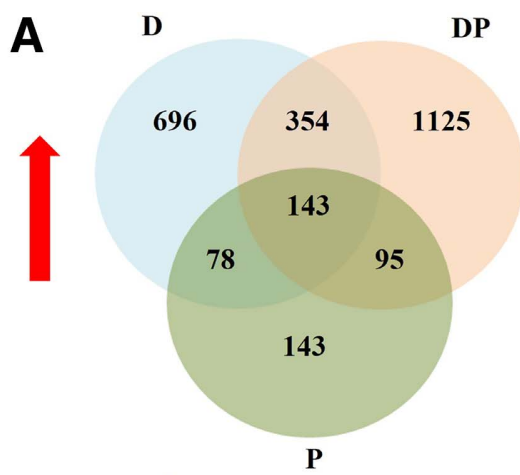

B

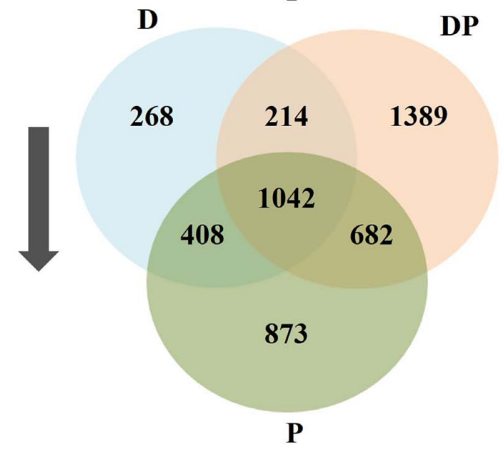

DW

UP

Plant-pathogen interaction

MAPK signalling

- Amino acid and nucleotide metabolism

Glycerolipid metabolism

Phosphatidylinositol signaling system

Linolenic acid metabolism

Galactose metabolism

Starch and sucrose metabolism

Phagosome

Arachidonic acid

Protein processing in ER

Protein export

Plant hormone signal transduction

Pentose phosphate pathway

Glutathione metabolism

Riboflavin metabolism

Isoflavonoid biosynthesis

Ribosome

Ribosome
Alanine metabolism

TCA cycle

Arginine and proline metabolism

Terpenoid biosynthesis

Cystenine and methinine metabolism

Glycolysis/Gluconeogenesis

Endocytosis

MAPK signalling

Phenylpropanoid biosynthesis

Amino acid biosynthesis

Carbon metabolism

Plant-pathogen interaction

Specialized metabolism

Metabolic pathway

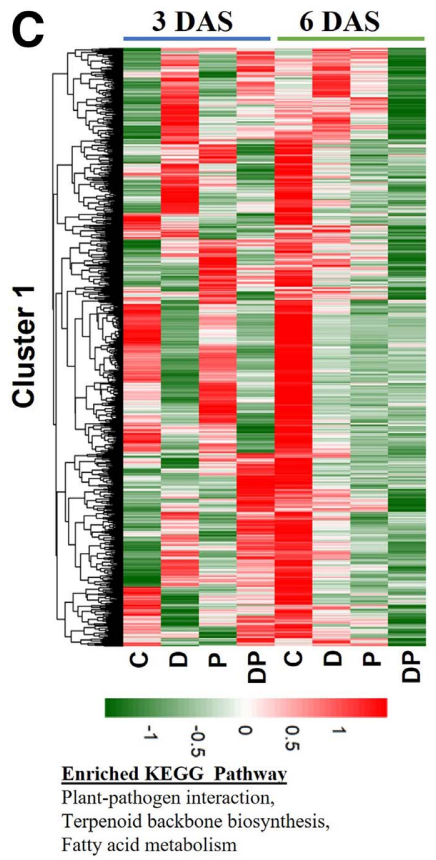

D

$\begin{array}{llllllllll}350 & 300 & 250 & 200 & 150 & 100 & 50 & 0 & 50 & 100\end{array}$

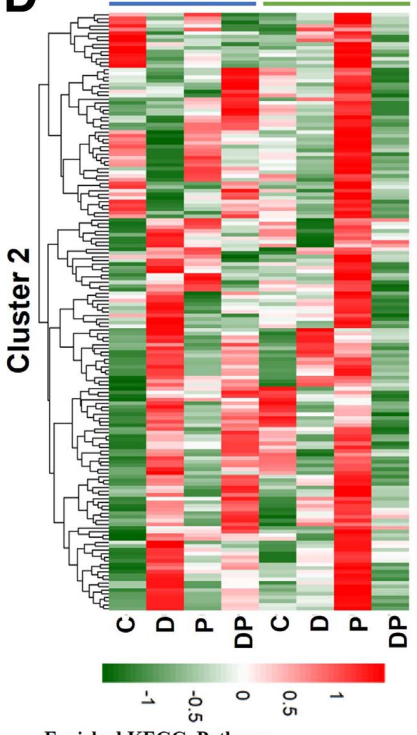

Enriched KEGG Pathway

Specialized Metabolism (Phenylpropanoid biosynthesis, and terpenoid biosynthesis) and primary metabolism.

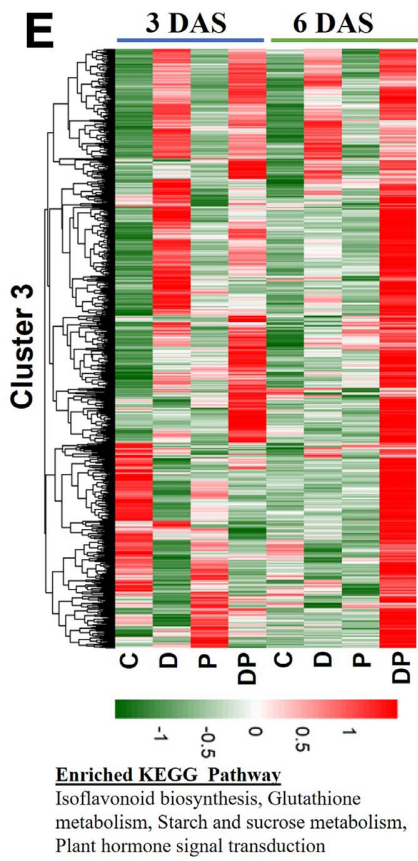

Fig. 3. Differential expression of genes under drought (D), pathogen (P), and pathogen-plus-drought (DP) treatments. A, Venn diagram illustrating the overlap of differentially expressed genes (DEGs) specifically upregulated and downregulated during combined drought and pathogen stress at 6 days after sowing (DAS). B, Functional enrichment of DEGs as upregulated (UP, yellow bar) and downregulated (DW, green bar) features distinct in P and DP stress at 6 DAS. Heat map showing the gene expression $\mathbf{C}$, cluster 1; D, cluster 2; and $\mathbf{E}$, cluster 3. Clusters were derived from weighted gene coexpression network analysis (WGCNA). WGCNA was performed using fragments per kilobase per million reads values of the genes. The enriched Kyoto Encyclopedia of Genes and Genomes (KEGG) pathway is listed below the corresponding clusters. All enriched pathways had a $P$ value $\leq 0.05$. C $=$ control. DEGs were obtained after comparing treatments with a control. 
under DP stress compared with the control, suggesting their role in reprogramming plant defense. Moreover, genes related to plasma membrane function (integrity) and cellular damage control (cell division, cell viability, and signal transduction) showed differential transcriptional regulation under DP stress at 6 DAS (Supplementary Fig. S24). For instance, the transcript level of the cytokinin oxidase $6(C K X 6)$ gene involved in cytokinin regulation and lateral root formation was reduced in infected roots
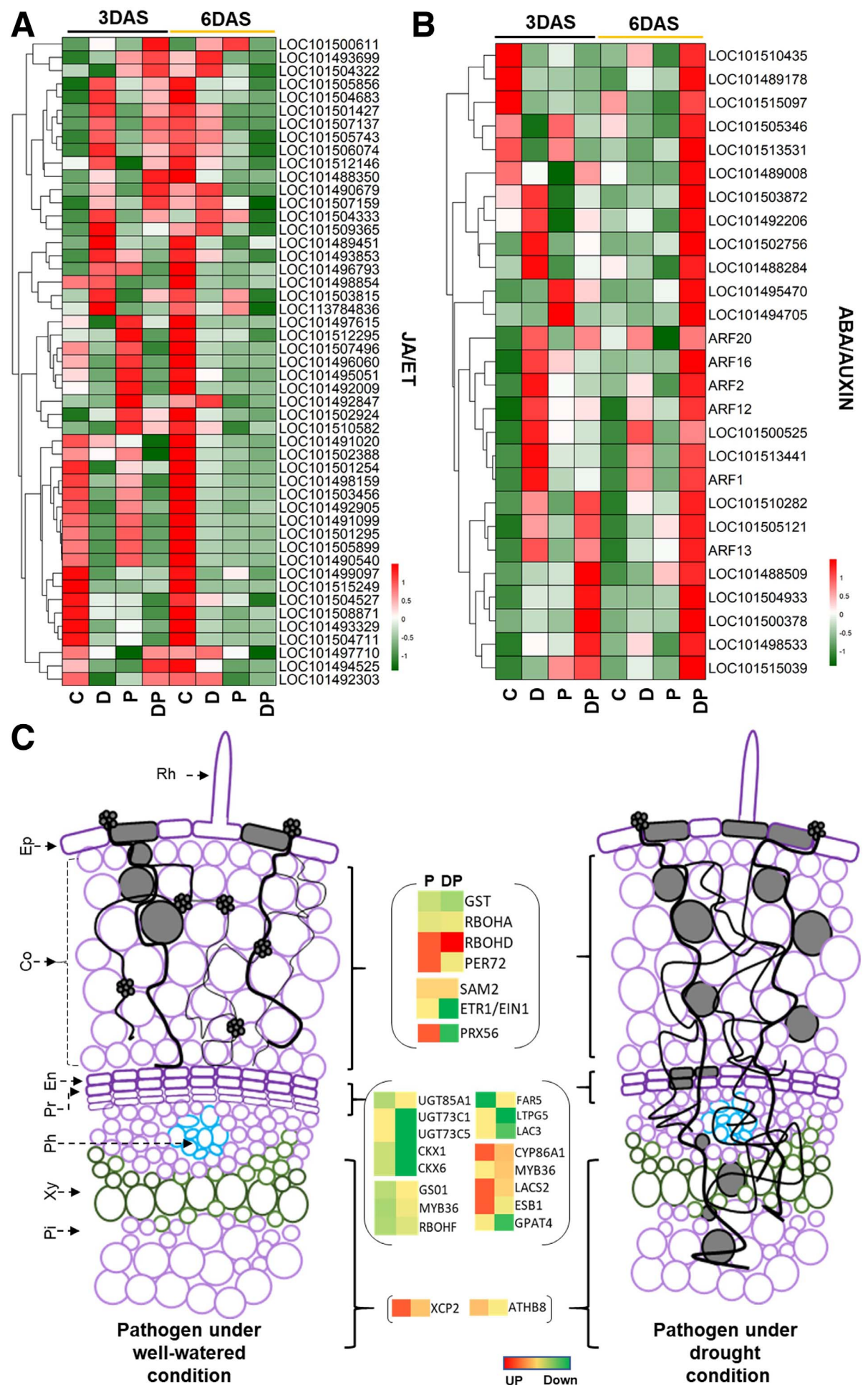

Fig. 4. Transcriptional reprogramming of hormone- and defense-related genes under pathogen and drought stress. A, Heat map showing the differentially expressed genes (DEGs) of jasmonic acid and ethylene (JA/ET) hormone regulation in drought (D), pathogen (P), and pathogen-plus-drought (DP) stress. B, Heat map showing the DEGs of abscisic acid and auxin hormone signaling under D, P, and DP treatments. Red represents an increase in transcript level and green represents a decrease in transcript level. C, A model depicting the differential expression of cell-layer-specific innate defense-related genes in P and DP stress treatments. The model was drawn based on a toluidine blue O-stained chickpea root. Pathogen-infected roots show fungal colonization up to the endodermal barrier layer, with the formation of daughter microsclerotia (diagram on the left). The restricted colonization in the cortex region is consistent with the upregulation of genes (MYB26, $E S B 1$, and $L A C 3$ ) involved in endodermal barrier reinforcement (Casparian strip and suberin). Pathogen-infected roots subjected to drought stress show extensive fungal colonization beyond the endodermal barrier (diagram on the right), consistent with the downregulation of genes (PER72, PRX56, and GST) involved in innate immunity defense. Abbreviations: $\mathrm{Rh}=$ root hair, $\mathrm{Ep}=$ epidermis, $\mathrm{Co}=$ cortex, $\mathrm{En}=$ endodermis, $\mathrm{Pr}=$ pericycle $, \mathrm{Ph}=\mathrm{phloem}, \mathrm{Xy}=\mathrm{xylem}$, and $\mathrm{Pi}=$ pith . 
under drought stress (Fig. 4B). CKX6 reduces the cytokinin level and is accordingly involved in cell division and lateral root formation. $C K X 6$ overexpression in the roots was shown to impart drought tolerance to chickpea plants (Khandal et al. 2020). This suggests that the reduced cytokinin level in chickpea roots might be conducive for the DRR fungus to colonize further. It is noteworthy that nodule-forming legume roots show characteristic cell division upon cytokinin treatment (Gauthier-Coles et al. 2019). Whether there is any connection between hyperplasia at the pericycle or endodermal region upon pathogen infection and cytokinin signaling is an open question. However, the transcript level of reactive oxygen species (ROS)-related genes involved in recognizing a pathogen by the host is increased by both the pathogen alone and DP stress (Fig. 4B). The induced expression of ROS genes in both individual and combined stress at 6 DAS is likely a response to the oxidative burst generated as a secondary response, which could be an additive effect of drought stress and DRR fungus infection.

Because drought stress changed the spatiotemporal growth and defense response of chickpea infected with DRR fungus, we further investigated whether this change in response also regulated root architecture, in terms of concentric cell layers, for enhanced susceptibility to DRR fungus. Infection with DRR fungus under well-watered conditions at 6 DAS induced the expression of celllayer-specific defense genes such as peroxidase 72 (PER72) in the cortex, long-chain acyl-CoA synthetase 2 (LACS2) in the endodermis, and cysteine protease (XCP2) in the stele (Fig. 4B). In contrast, these genes were downregulated in infected roots subjected to drought stress. The Casparian strip and suberin depositions are well-known endodermal barriers against pathogen attacks (Fröschel et al. 2021). Interestingly, Casparian strip formation and suberin biosynthesis genes such as enhanced suberin 1 (ESB1), lipid transfer protein (LTPG5), and hydroxylase of root-suberized tissue (HORST/CYP86Al) were coordinately upregulated under the pathogen plus well-watered treatment at 6 DAS; however, a remarkable downregulation of these genes was observed under DP stress at 6 DAS (Fig. 4B). Thus, drought changed the spatiotemporal dynamics of growth, defense, and metabolic pathway genes, which perform differential roles in chickpea immunity, survival, and fitness under pathogen attack. Most defense genes involved in innate immunity were downregulated in infected roots upon drought stress imposition. Due to the extensive transcriptional reprogramming under the influence of combined drought and pathogen stress, it is anticipated that DRR pathogen infection weakens the endodermal barrier by reducing the translation of genes involved in the endodermal barrier enforcement for extensive colonization.

The current study highlights that the rate and extent of DRR fungal infection and colonization are slow in adequately irrigated chickpea plants but, in contrast, are accelerated under droughtstress conditions (Supplementary Figs. S25 to S28). We systematically examined the role of drought stress in DRR disease aggravation using detailed microscopy analysis. Our RNA-seq data suggest that the downregulation of genes involved in ROS, endodermal cell layer formation, and JA and ET hormone regulation dampened the plant's defense response mechanisms upon drought stress imposition. We also showed that plants with high lateral root density possess tolerance to soilborne pathogen infection and drought stress (Pandey et al. 2017). At advanced infection stages, pathogen-only-treated plants had more lateral roots, with the emergence of new lateral roots. In contrast, plants exposed to pathogen and drought stress had fewer lateral roots (Supplementary Figs. S25 to S27). CKX6, responsible for lateral root formation, was downregulated under drought stress.

This study provides preliminary answers to key questions about how drought weakens the innate immunity in the chickpea-DRR system. It also reveals the component of plant defense at the molecular level and identifies the chief factors participating in the predisposition to infection and subsequent disease severity.

\section{MATERIALS AND METHODS}

\section{Plant growth conditions.}

Chickpea plants (genotype JG 62, also known as ICC 4951) were grown in Soilrite potting medium (Soilrite mix; Keltech Energies Ltd., Bangalore, India) and maintained in the growth room at a room temperature of $26 \pm 2^{\circ} \mathrm{C}$, a 16-h photoperiod with a light intensity of $150 \mu \mathrm{mol} \mathrm{m} \mathrm{m}^{-2} \mathrm{~s}^{-1}$, and relative humidity of $70 \%$. Unless another genotype name was specified, this genotype was used in the experiments.

\section{Pathogen inoculation, drought, and combined stress imposition using blotting paper technique.}

The blotting paper technique demonstrated by Irulappan and Senthil-Kumar (2021) was used in the study. Briefly, surfacesterilized chickpea seeds were sown, and a nursery was prepared. Five-day-old plants were uprooted, washed, and used for the infection assay. DRR fungal inoculum (ITCC 8635, NCBI GenBank ID MH509971.1) was prepared by inoculating $500 \mathrm{ml}$ of potato dextrose broth with a fungal agar plug from a pure slant culture. Fungal propagules (both mycelia and microsclerotia) from a fiveday-old liquid fungal culture were filtered out, washed, and used to infect the plant roots. Uprooted plants were placed on wet blotting paper, and fungal propagules were kept on the top side of the root. At 6 and 10 DAI, trays were separated for control, drought, pathogen, and DP (combined stress) treatments. For control and pathogen treatments, the wet weight of the blotting paper ( 2 by $2 \mathrm{~cm}$ ) was $112 \pm 3 \mathrm{mg}$ (100\% water content). For drought and DP stress treatments, the weight of blotting paper $(2$ by $2 \mathrm{~cm})$ was $70 \pm 6 \mathrm{mg}$ (37.5\% decrease in water content). Three days after drought imposition, root water potential was measured using automated soil and leaf water potential system (PSYPRO; Wescor Inc., Logan, UT, U.S.A.). The water potential of well-watered and drought-stressed roots was -0.1 and $-0.8 \mathrm{MPa}$, respectively.

\section{Pathogen infection, drought, and combined stress imposition using sick pot technique.}

Soilrite medium with fungal pathogen inoculum was used to prepare the sick pot experiment (Irulappan and Senthil-Kumar 2021). The 5\% (wt/wt) dry weight of the inoculum was used for disease progression assays. Soilrite medium without fungus was used for control and drought-only stress treatments. Control and pathogen-only treatments were maintained at the pot mixture water status of $90 \%$ field capacity (FC). Drought stress was imposed by withholding the watering by the gravimetric method (Sinha et al. 2019). For the drought-only and combined DP treatment, $35 \% \mathrm{FC}$ for 5 days was considered.

\section{In planta fungal pathogen quantification by quantitative PCR.}

Root samples infected with DRR fungal propagules from blotting paper and sick pot experiments were collected every day starting 1 DAI (Irulappan and Senthil-Kumar 2021; Sinha et al. 2019) to 9 DAI and immediately frozen in liquid $\mathrm{N}_{2}$ and stored at $-80^{\circ} \mathrm{C}$. Total DNA was isolated using the cetyltrimethylammonium bromide method (Springer 2010). The DNA quantity was measured using a NanoDrop Spectrophotometer (Thermo Fisher Scientific, Waltham, MA, U.S.A.). For fungal DNA quantification from the samples, a standard curve was developed using the known concentrations of fungal DNA $(0.001,0.01,0.1,1,10$, and $100 \mathrm{ng}$ ) (Irulappan et al. 2021). In all, $50 \mathrm{ng}$ of template DNA from the test samples was used for quantitative PCR. The in planta fungal DNA quantity was calculated by using the standard curve. 


\section{Confocal microscopy.}

Root samples collected from pathogen and combined stress treatments were fixed in $10 \%$ neutral buffered formalin followed by vacuum infiltration. Samples were washed with RO water thrice for $10 \mathrm{~min}$ each, and an agar block (4\% agar) was made. Transverse sections (TS) $(100 \mu \mathrm{m})$ were made using a vibrating blade microtome (Leica VT1000 S; Leica Biosystems, Mumbai, India) and cleared in $10 \% \mathrm{KOH}$ for $8 \mathrm{~h}$ in a rotary shaker (50 rpm). Then, $\mathrm{KOH}$ was replaced with $1 \mathrm{M}$ Tris-HCL ( $\mathrm{pH} 8.0$ ) for $1 \mathrm{~min}$, and sections were maintained in $50 \mathrm{mM}$ Tris-HCL (pH 8.0). WGA-FITC (L4895; Sigma-Aldrich Chemicals Private Limited, Bangalore, India) and calcofluor white 2MR (CFW 2MR) (18909; Sigma-Aldrich Chemicals Private Limited) stains were added over the TS sections placed on a microscope slide followed by placement of a coverslip. The microscopy observations were performed under a $\times 20$ objective lens of a confocal microscope (Leica TCS SP5/SP8; Leica Biosystems) with excitation and emission bandpass of 495 to $515 \mathrm{~nm}$ for WGA-FITC and 350 to 432 for CFW.

\section{Scanning electron microscopy.}

Root samples were collected at 1 DAI (Irulappan and SenthilKumar 2021) to $5 \mathrm{DAI}$ and fixed in $2.5 \%$ glutaraldehyde in $0.1 \mathrm{M}$ sodium phosphate buffer ( $\mathrm{pH}$ 7.2) by vacuum infiltration. Then, roots were dehydrated in acetone series $(30,50,70,80,90,95$, and $100 \%$ ) twice at each concentration for $10 \mathrm{~min}$. In the end, roots were kept in $100 \%$ acetone for $2 \mathrm{~h}$. Roots were subjected to a critical point dryer (Leica EM CPD300; Leica Biosystems). Images were captured using a scanning electron microscope (Carl-Zeiss-Strasse, Oberkochen, Germany) at $\times 100, \times 250$, and $\times 500$ magnifications.

\section{RNA-seq library preparation.}

Libraries were prepared in three biological replicates using the Breath Adapter Directional sequencing (BrAD-seq) method (Townsley et al. 2015). Strand-specific mRNA libraries were made using the YourSeq full-transcript RNA-seq library protocol per the manufacturer's instructions (Amaryllis Nucleics Biotechnology Company, Oakland, CA, U.S.A.). These RNA-seq libraries were sequenced on the Illumina NOVASEQ6000 platform (Illumina Way, San Diego, CA, U.S.A.) and reads were generated in 150-bp paired-end format.

\section{RNA-seq data processing.}

The quality of raw sequences was evaluated using FastQC (version 0.11 .5 ). Low-quality reads with a Phred score $<20$ were filtered, and adapters were removed using Trimmomatic version 0.39 (Bolger et al. 2014). Reads that passed quality filtering criteria were aligned to the chickpea genome of CDC Frontier, a Canadian Kabuli chickpea variety (Varshney et al. 2013) using HISAT2 version 2.1.0 (Kim et al. 2015) using default parameters. The aligned reads were used for expected number of fragments per kilobase per million reads (FPKM) calculation using Stringtie (Pertea et al. 2015). Differential gene expression was determined by using Cuffdiff (Trapnell et al. 2012). The transcripts with $\log _{2^{-}}$ fold change $\geq 1$ (upregulated genes) or $\leq-1$ (downregulated genes) with q-value cutoff $\leq 0.05$ were considered as differentially expressed. PCA was performed on normalized FPKM values using the opls function of the $\mathrm{R}$ package ropls (Thévenot et al. 2015).

\section{GO, Mapman, and KEGG enrichment analysis.}

Mapman annotations created with Mercator4 version 3.0 were used to analyze metabolic pathways of DEGs (Schwacke et al. 2019). Then, the enriched Mapman pathways were identified using a hypergeometric test $(P$ value $\leq 0.05)$. Additionally, the KOBASi software was used to determine the statistical significance of
KEGG pathways for DEGs with a $P$ value of $0.05(\mathrm{Bu}$ et al. 2021). To identify Arabidopsis orthologs, we blasted chickpea proteins against the Arabidopsis proteome TAIR database. The first five hits with percent similarity higher than $30 \%$, coverage $\geq 30 \%$, e-value 1e-10, and score cut-off of 100 were reverse blasted against the chickpea proteome. Then, using TAIR IDs as a query for agriGO, $\mathrm{GO}$ analysis was performed.

\section{WGCNA analysis.}

The coexpression module of DEGs was analyzed using the $\mathrm{R}$ package WGCNA (version 1.61). The modules were generated using the default settings, with the exception that the power was set to 20 , the TOMType was assigned, the minModuleSize was set to 30 , and the merge CutHeight was set to 0.25 .

\section{Statistical analysis.}

The significance of the data was analyzed using one-way analysis of variance (ANOVA) or two-way ANOVA followed by Tukey's multiple comparisons test or Holm-Sidak's multiple comparisons test, with an asterisk indicating the level of significance in GraphPad Prism version 6.0.0 for Windows (GraphPad Software, San Diego, CA, U.S.A.).

\section{Data availability.}

The RNA-seq data can be accessed from BioProject (ID number PRJNA749609).

\section{ACKNOWLEDGMENTS}

We thank the confocal microscopy facility, scanning electron microscopy facility, and central instrument facility at the National Institute of Plant Genome Research (NIPGR); the Department of Biotechnology, India, for use of the Nikon-SMZ25 stereo fluorescence microscope (BT/PR23489/BPA/ 118/287/2017); the the eLibrary Consortium (DeLCON), India, for access to e-resources; R. H. Tarafdar and S. Badaik (NIPGR) for field assistance; K. S. Rishika, K. Jaya Chendrayan, and A. Durgadevi for their technical assistance; NIPGR Plant Growth Facility for providing growth space and maintenance; and B. Longchar, M. Patil, A., R. Mirchandani, and A. Singh at NIPGR for internally reviewing the manuscript and scrutinizing the raw data.

\section{AUTHOR-RECOMMENDED INTERNET RESOURCES}

\section{FastQC version 0.11.5:}

https://www.bioinformatics.babraham.ac.uk/projects/fastqc/

ITCC 8635 :

https://iari.res.in/index.php?option=com_content\&view=article \&id=175\& Itemid $=520$

R package WGCNA version 1.61:

https://cran.r-project.org/web/packages/WGCNA/index.html

\section{LITERATURE CITED}

Ayliffe, M., Periyannan, S. K., Feechan, A., Dry, I., Schumann, U., Wang, M. B., Pryor, A., and Lagudah, E. 2013. A simple method for comparing fungal biomass in infected plant tissues. Mol. PlantMicrobe Interact. 26:658-667.

Barberon, M. 2017. The endodermis as a checkpoint for nutrients. New Phytol. 213:1604-1610.

Bolger, A. M., Lohse, M., and Usadel, B. 2014. Trimmomatic: A flexible trimmer for Illumina sequence data. Bioinformatics 30:2114-2120.

Bu, D., Luo, H., Huo, P., Wang, Z., Zhang, S., He, Z., Wu, Y., Zhao, L., Liu, J., Guo, J., Fang, S., Cao, W., Yi, L., Zhao, Y., and Kong, L. 2021. KOBAS-i: Intelligent prioritization and exploratory visualization of biological functions for gene enrichment analysis. Nucleic Acids Res. 49:W317-W325.

Chandran, U. S., Tarafdar, A., Mahesha, H. S., and Sharma, M. 2021. Temperature and soil moisture stress modulates the host defense response in chickpea during dry root rot incidence. Front. Plant Sci. 12:932.

Chowdhury, S., Basu, A., and Kundu, S. 2017. Biotrophy-necrotrophy switch in pathogen evoke differential response in resistant and susceptible sesame involving multiple signaling pathways at different phases. Sci. Rep. 7:1-17. 
Fröschel, C., Komorek, J., Attard, A., Marsell, A., Lopez-Arboleda W. A., Le Berre, J., Wolf, E., Geldner, N., Waller, F., Korte, A., and Dröge-Laser, W. 2021. Plant roots employ cell-layer-specific programs to respond to pathogenic and beneficial microbes. Cell Host Microbe 29:299-310.e7.

Gauthier-Coles, C., White, R. G., and Mathesius, U. 2019. Nodulating legumes are distinguished by a sensitivity to cytokinin in the root cortex leading to pseudonodule development. Front. Plant Sci. 9:1901.

Geldner, N. 2013. The endodermis. Annu. Rev. Plant Biol. 64:531-558.

Ghosh, R., Sharma, M., Telangre, R., and Pande, S. 2013. Occurrence and distribution of chickpea diseases in central and southern parts of India. Am. J. Plant Sci. 4:940-944.

Infantino, A., Kharrat, M., Riccioni, L., Coyne, C. J., McPhee, K. E., and Grünwald, N. J. 2006. Screening techniques and sources of resistance to root diseases in cool season food legumes. Euphytica 147:201-221.

Irulappan, V., Mali, K. V., Patil, B. S., Manjunatha, H., Muhammad, S., and Senthil-Kumar, M. 2021. A sick plot-based protocol for dry root rot disease assessment in field-grown chickpea plants. Appl. Plant Sci. 9:e11445.

Irulappan, V., and Senthil-Kumar, M. 2021. Dry root rot disease assays in chickpea: A detailed methodology. J. Vis. Exp. 167:e61702.

Ivanov, S., and Harrison, M. J. 2014. A set of fluorescent protein-based markers expressed from constitutive and arbuscular mycorrhizainducible promoters to label organelles, membranes and cytoskeletal elements in Medicago truncatula. Plant J. 80:1151-1163.

Karadi, A., Samineni, S., Sajja, S., Sharma, M., Thudi, M., Mallikarjuna, B. P., Viswanatha, K. P., Varshney, R. K., and Gaur, P. M. 2021. Molecular mapping of dry root rot resistance genes in chickpea (Cicer arietinum L.). Euphytica 217:123.

Khandal, H., Gupta, S. K., Dwivedi, V., Mandal, D., Sharma, N. K., Vishwakarma, N. K., Pal, L., Choudhary, M., Francis, A., Malakar, P., and Singh, N. P. 2020. Root-specific expression of chickpea cytokinin oxidase/dehydrogenase 6 leads to enhanced root growth, drought tolerance and yield without compromising nodulation. Plant Biotechnol. J. 18:2225-2240.

Kieber, J. J. 1997. The ethylene signal transduction pathway in Arabidopsis. J. Exp. Bot. 48:211-218.

Kim, D., Langmead, B., and Salzberg, S. L. 2015. HISAT: A fast spliced aligner with low memory requirements. Nat. Methods 12:357-360.

Mandal, D., and Sinharoy, S. 2019. A toolbox for nodule development studies in chickpea: A hairy-root transformation protocol and an efficient laboratory strain of Mesorhizobium sp. Mol. Plant-Microbe Interact. 32:367-378.

Maqbool, M. A., Aslam, M., and Ali, H. 2017. Breeding for improved drought tolerance in chickpea (Cicer arietinum L.). Plant Breed. 136:300-318.

Nene, Y. L., Haware, M. P., and Reddy, M. V. 1981. Chickpea diseases: Resistance-screening techniques. International Crops Research Institute for the Semi-Arid Tropics, Patancheru, India.

Pandey, P., Irulappan, V., Bagavathiannan, M. V., and Senthil-Kumar, M. 2017. Impact of combined abiotic and biotic stresses on plant growth and avenues for crop improvement by exploiting physiomorphological traits. Front. Plant Sci. 8:537.

Pertea, M., Pertea, G. M., Antonescu, C. M., Chang, T. C., Mendell, J. T., and Salzberg, S. L. 2015. StringTie enables improved reconstruction of a transcriptome from RNA-seq reads. Nat. Biotechnol. 33:290295.

Rao, P. A., and Haware, M. P. 1987. Inheritance of dry root rot (Rhizoctonia bataticola) resistance in chickpea (Cicer arietinum). Plant Breed. 98:349352.

Schwacke, R., Ponce-Soto, G. Y., Krause, K., Bolger, A. M., Arsova, B., Hallab, A., Gruden, K., Stitt, M., Bolger, M. E., and Usadel, B. 2019. MapMan4: A refined protein classification and annotation framework applicable to multi-omics data analysis. Mol. Plant 12:879-892.

Sharma, M., and Pande, S. 2013. Unravelling effects of temperature and soil moisture stress response on development of dry root rot [Rhizoctonia bataticola (Taub.)] Butler in Chickpea. Am. J. Plant Sci. 4:584589.

Sinha, R., Irulappan, V., Mohan-Raju, B., Suganthi, A., and Senthil-Kumar, M. 2019. Impact of drought stress on simultaneously occurring pathogen infection in field-grown chickpea. Sci. Rep. 9:5577.

Sinha, R., Irulappan, V., Patil, B. S., Reddy, P. C. O., Ramegowda, V., Mohan-Raju, B., Rangappa, K., Singh, H. K., Bhartiya, S., and Senthil-Kumar, M. 2021. Low soil moisture predisposes field-grown chickpea plants to dry root rot disease: Evidence from simulation modeling and correlation analysis. Sci. Rep. 11:6568.

Springer, N. M. 2010. Isolation of plant DNA for PCR and genotyping using organic extraction and CTAB. Cold Spring Harb. Protoc. 2010: pp.pdb-prot5515.

Talekar, S. C., Lohithaswa, H. C., and Viswanatha, K. P. 2017. Identification of resistant sources and DNA markers linked to genomic region conferring dry root rot resistance in chickpea (Cicer arietinum L.). Plant Breed. 136:161-166.

Thévenot, E. A., Roux, A., Xu, Y., Ezan, E., and Junot, C. 2015. Analysis of the human adult urinary metabolome variations with age, body mass index, and gender by implementing a comprehensive workflow for univariate and OPLS statistical analyses. J. Proteome Res. 14: 3322-3335.

Townsley, B. T., Covington, M. F., Ichihashi, Y., Zumstein, K., and Sinha, N. R. 2015. BrAD-seq: Breath Adapter Directional sequencing: A streamlined, ultra-simple and fast library preparation protocol for strand specific mRNA library construction. Front. Plant Sci. 6: 366.

Trapnell, C., Roberts, A., Goff, L., Pertea, G., Kim, D., Kelley, D. R., Pimentel, H., Salzberg, S. L., Rinn, J. L., and Pachter, L. 2012. Differential gene and transcript expression analysis of RNA-seq experiments with TopHat and Cufflinks. Nat. Protoc. 7:562-578

Varshney, R. K., Song, C., Saxena, R. K., Azam, S., Yu, S., Sharpe, A. G., Cannon, S., Baek, J., Rosen, B. D., Tar'an, B., and Millan, T. 2013. Draft genome sequence of chickpea (Cicer arietinum) provides a resource for trait improvement. Nat. Biotechnol. 31:240-246. 